\title{
Radio Resource Application in Aeronautical Mobile Communication System
}

\author{
Fan $\mathrm{Hu}^{\mathrm{a}}$, Tao Zhang ${ }^{\mathrm{b}}$ \\ School of Electronic and Information Engineering, Beihang University \\ Beijing Laboratory for General Aviation Technology \\ Beijing, 100191, P.R.China \\ ahufan@buaa.edu.cn, bzhtao@buaa.edu.cn
}

Keywords: Aeronautical mobile communication, Resource application, Smoothing factor

\begin{abstract}
This paper systematically analyzed resource application allocation process and the timing relationship of resource application in aeronautical mobile communication system's link layer. This paper aimed at a new generation space ground data link technology which was called L-band digital aeronautical communication system type-1 (LDACS1). Based on the traditional traffic predictionbased resource application algorithm, this paper proposed an algorithm based on the combination of feedback control and prediction of exponential smoothing, to compensate for the lack of accuracy of resource application amount in existing algorithms of forecasting. The algorithm introduced smoothing factor to make the application bandwidth smooth. Through reducing the volatility of applications, the algorithm will improve the the difference between the amount of application and allocation and reduce fluctuations in the queue which caused by inaccurate application prediction, thereby reducing the time delay. The simulation results show that the performance of the algorithm is much better than the available resource application algorithms. Under the environment of different degrees of load, this algorithm can reduce time delay and packet loss rate.
\end{abstract}

\section{Introduction}

Digital broadband data link technology between the space and ground in aeronautical mobile communication system is one of the key technologies to achieve the interconnection and interworking between plane and ground during the flight ${ }^{[1]}$. Digital broadband data link between the space and ground technology at link layer need to solve the problem of multiple planes, a variety of business sharing limited radio resource between space and ground. In this respect, using mechanism of bandwidth dynamic allocation on demand to solve the contradiction between resource utilization and service quality is an important research aspect.

In order to support advanced air traffic management business in the future better, the European Space Agency put forward a L-band digital aeronautical communication system type-1 (LDACS1) ${ }^{[2][3]}$ based on resource application allocation mechanism.

In the mechanism of bandwidth dynamic allocation on demand, users send bandwidth request according to the actual need, then the resource manager allocate bandwidth for users according to its bandwidth request to deal with the high burst in multimedia services and improve the utilization of radio resource ${ }^{[4][5]}$. High-bandwidth applications will reduce system efficiency, low-bandwidth applications will reduce the service quality. So the accuracy of application amount is very important in this mechanism.

However, the existing protocols for wireless resource application study is still less. Literature [6][7] considered that when calculating the application bandwidth, the amount of business arriving from mobile node sending resource application information to base station receiving the information is not zero. The traffic forecasting in resource application made up the defect of thoughts that the arrival quantity is zero. Literature [8] did a business forecast first, then it considered that the allocation amount is equal to the application amount which solved the disadvantage of repeated application. But actually application and allocation amount is not equal. 
This paper systematically analyzed the resource application allocation process in link layer of data link network between the space and ground and studied the timing relationship in resource application allocation process. Combining with the long delay in network between the space and ground, it proposes a new algorithm of resource application based on feedback control and exponential smoothing predict. Predictive value of application is decided by queue length in this algorithm. Hypothesis allocation amount is equal to application amount. After business forecasting and allocation amount forecasting, this algorithm adjusted the difference between application amount and queue length to make the difference smaller. Finally volatility of application amount decreased and the difference between the application and the actual queue length decreased and the forecast of application amount is more accurate.

\section{Resource Application Allocation Algorithm}

Bandwidth application allocation mechanism of data link between the space and ground consists of two parts: the aeronautical mobile node and the base station. Its timing chart $^{[2][3][9][10]}$ of running process is shown in Fig.1.

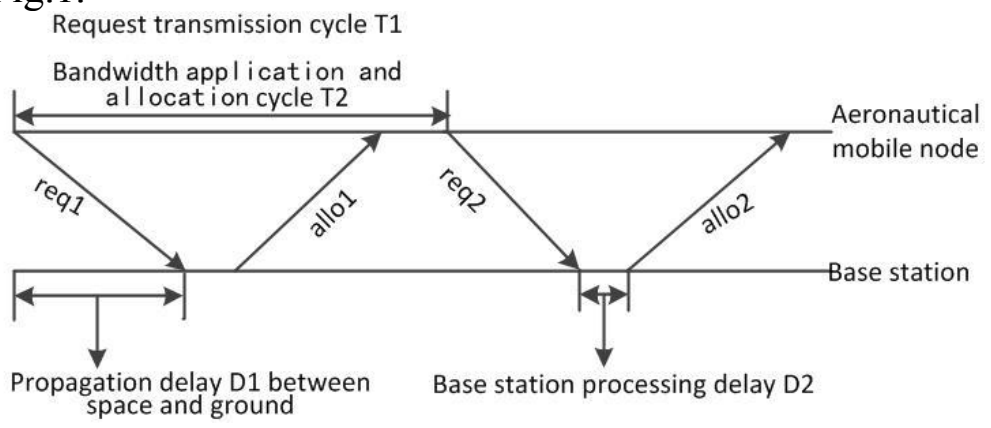

Fig. 1. Bandwidth application allocation process

Aeronautical mobile node (hereinafter referred to as plane) sends bandwidth request req at the beginning of each multiframe. They reach the ground terminal after a propagation delay between the space and ground D1. Ground terminal allocates resource after counting all applications and send allocation results to plane after D2. At last, plane transmits data.

Fig. 1 shows that the bandwidth application allocation process of data link between space and ground has delay characteristics. Applied resource cannot be allocated timely and the current packet cannot be transmitted. It will be transmitted in the next frame. The purpose of the bandwidth application is sending data out. Therefore the amount of resource application is determined by the amount of cached data. The current application amount should be the amount of accumulated data at the time of next frame to obtain appropriate resource.

Qin Yong ${ }^{[8]}$ generated a bandwidth request based on the length of queue and the predictive value of data amount reached in the next frame. Combining with feedback control, he generated a bandwidth request which is calculated as follows:

$$
r_{r e q}^{n}(t)=\left[q_{r}(\mathrm{t})-\int_{t-T_{s f}}^{t} r_{r e q}^{n}(\tau) d_{\tau}\right]^{+}+\int_{t}^{t+T_{s f}} r_{i n}^{n}(\tau) d_{\tau}
$$

The bandwidth request of the terminal $\mathrm{n}$ in the time $\mathrm{t}$ is $r_{r e q}^{n}(\mathrm{t}) \cdot q_{r}(\mathrm{t})$ is the queue length at $\mathrm{t}$. $T_{s f}$ is the frame period. $\int_{t-T_{s f}}^{t} r_{r e q}^{n}(\tau) d_{\tau}$ is the application amount of last frame. $\int_{t}^{t+T_{s f}} r_{i n}^{n}(\tau) d_{\tau}$ is the predictive value of data amount reached in the next frame. The above-mentioned algorithm of bandwidth application allocation used the combination of feedback control and linear prediction to predict queue length of the next frame. Compared to the method whose application is only based on current queue length, this algorithm can effectively control the queue length and delay. But the amount of application may not be equal to allocation, so there will be an error in the queue length's estimate of the next frame and queue length will fluctuate greatly. This will lead a consequence that the queue length is larger than the maximum value in several times and the packet loss rate is high. The packet loss process is shown in Fig.2. 


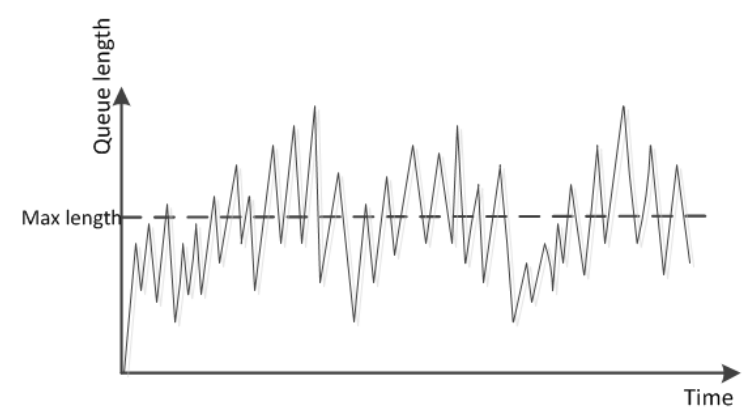

Fig. 2. Packet loss process

Liu Lei ${ }^{[11]}$ and others improved the formula on the basis of (1).Aiming at the effect of freedom allocation of remaining bandwidth on the feedback control, they put the feedback control on the base station and present a new application allocation algorithm based on base station's feedback control and prediction:

$$
\begin{aligned}
& r_{r e q}^{n}\left(t+\frac{T_{s f}}{2}\right)=\left[q_{r}(\mathrm{t})-\int_{t-T_{s f}}^{t} a_{\text {assign }}(\tau) d_{\tau}+\int_{t}^{t+T_{s f}} r_{i n}^{n}(\tau) d_{\tau}\right]^{+} . \\
& a_{\text {assign }}^{n}\left(t+T_{s f} / 2\right)=\frac{r_{r e q}^{n}\left(t+T_{s f} / 2\right)}{\sum_{1}^{N} r_{r e q}^{n}\left(t+T_{s f} / 2\right)} * C .
\end{aligned}
$$

Base station generates actual bandwidth request $r_{r e q}^{n}\left(\mathrm{t}+T_{s f} / 2\right)$ on the time $\mathrm{t}+T_{s f} / 2$ based on all the terminals' bandwidth requests $r_{r e q}^{n}(\mathrm{t})$ and distribution results in the last application allocation cycle and allocate bandwidth according to (3). $\mathrm{C}$ is total bandwidth. $\mathrm{N}$ is total amount of terminal.

The algorithm can predict the application of next frame more accurately to maximize reduce queue fluctuations and operational delay. But this method does not consider the impact caused by large number of packets on service quality. When business volume is larger than the average, application will fluctuate greatly and result in substantial growth delay. The time delay is shown in Fig.3.

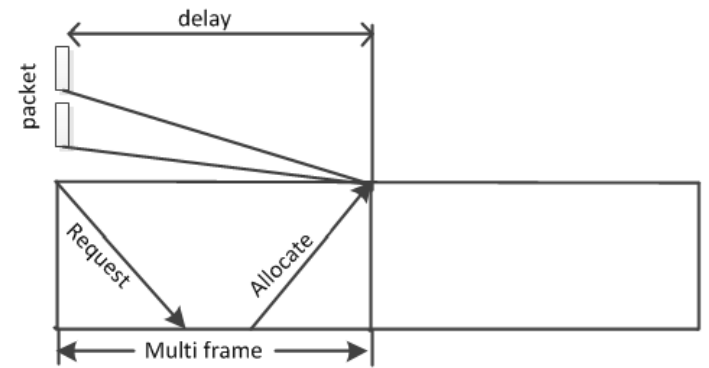

Fig. 3. Time delay caused by resource application and allocation

\section{Bandwidth Application Algorithm Based on Feedback Control and Exponential Smoothing Prediction}

In the link of LDACS1, frame structure in data link between space and ground ${ }^{[2][3][9][10]}$ is shown in Fig.4. DC frame in Plane generates a bandwidth request and sends it to base station. CC frame in base station allocate resource after integration of all bandwidth applications. When planes receive the allocation results, they start transmitting data through data frame. This process represents that application allocation interval is a DC frame period.

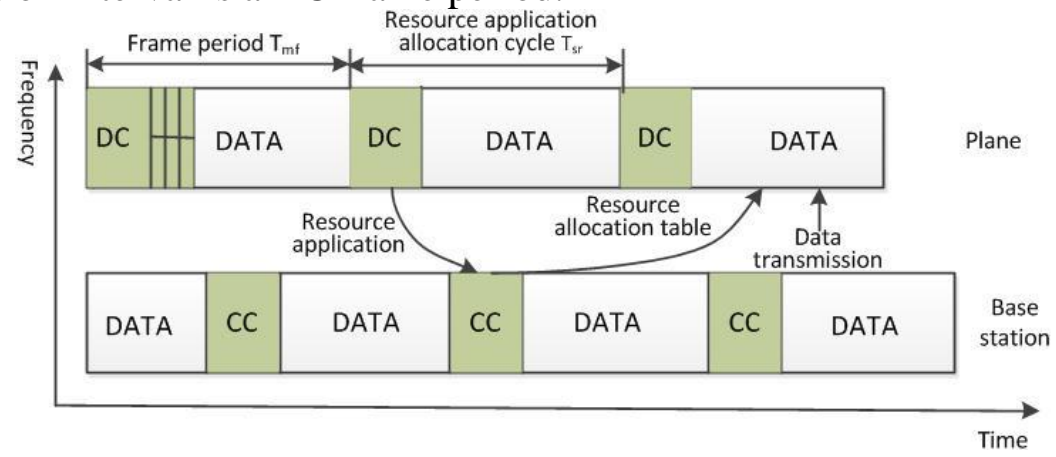

Fig. 4. Frame structure of LDACS1 
To simplify the complexity of data transformation in application allocation process, this article simplified the amount of data as the number of data packets. Queue length is also counted according to the number of packets.

Formula (1) simplified as:

$\mathrm{s}(\mathrm{t})=\mathrm{y}(\mathrm{t})-\mathrm{s}(\mathrm{t}-1)+\operatorname{arr}$.

The equation aims at one of the plane users. It omitted the $n$ parameters. In the application allocation process, there are many users and system has limited resource. Radio resource applications reach the base station and enter the buffer queue. Radio resource management unit judges whether total resource application is greater than the remaining bandwidth: if total resource application is greater than the remaining bandwidth, base station will meet each user's request. $a(t-1)=s(t-1)$. a $(t-$ 1 ) is the amount of allocation at time $t-1$. If bandwidth is not adequate, the resource is distributed pro rata.

The situation that total resource application is less than the remaining bandwidth will appear. The feedback control process is shown in Fig.5. If a $(\mathrm{t}-1)<\mathrm{s}(\mathrm{t}-1), \mathrm{s}(\mathrm{t})<\mathrm{y}(\mathrm{t}+1)$. It represents that the amount of allocation at $t$ is less than the amount user needs at $t+1$ which makes queue length increase at $t+2$. Then the feedback control plays a role. Without considering the accuracy of the amount of arrival, plane will generation a large number of application bandwidth at $t+2$ and receive a large number of resources. With the feedback control, although queue length increases at $t+2$, the queue length will reduce at $t+4$ because the amount of resource allocation at $t+2$ is larger than the amount of data arrival at $t+3$. Starting from $t$, the feedback continues to advance. If the amount of application is still greater than allocation at $t+1$, at this time the application distribution status is the same as $t$. Accumulative effect of twice state will cause the queue length and delay to increase significantly.

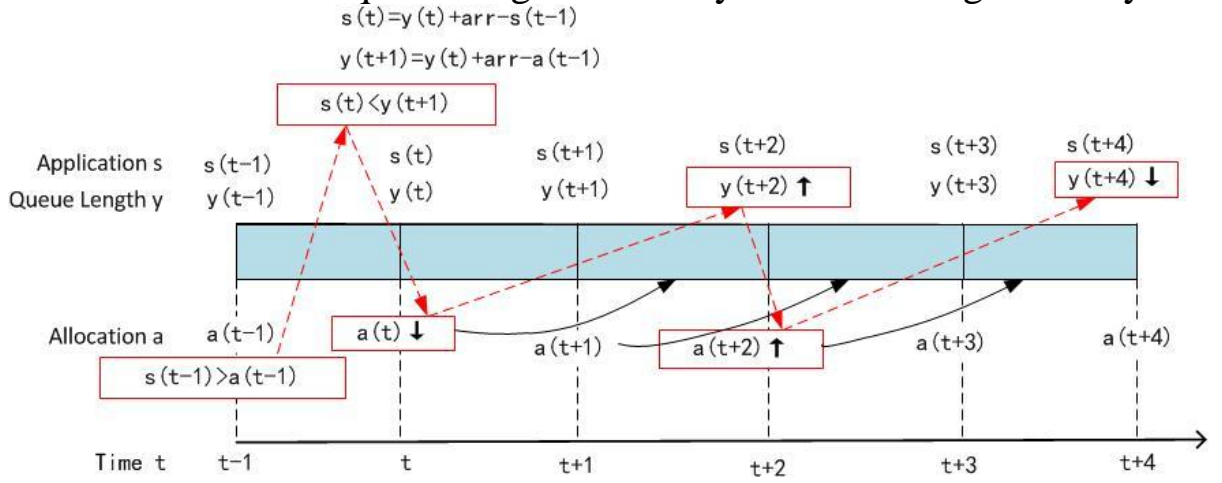

Fig. 5. Feedback control process

In the above process, the amount of incoming packets continuously greater than the average amount within a certain period of time, applications cannot be met. The cumulative effect leads to a substantial increase in queue length. The feedback control will make the queue length drop significantly. This makes queue length fluctuate greatly. Thus, the bigger the difference exists between application and allocation, the heavier the application fluctuates. This results in the instability of queue length and low efficiency of the system. The smoother the application is, the more stable queue is and the higher efficiency of the system is. Associating with linear exponential smoothing model, this paper proposes a method of application based on feedback control and prediction of linear exponential smoothing.

Exponential smoothing can predict future of events. The principle is that any one of exponential smoothing values is the weighted average value of the current value and exponential smoothing value of the previous period. Linear exponential smoothing prediction formula:

$\mathrm{s}(\mathrm{t})=\mathrm{a} * \mathrm{y}(\mathrm{t}-1)+(1-\mathrm{a}) * \mathrm{~s}(\mathrm{t}-1)$.

$\mathrm{s}(\mathrm{t})$ is the estimated value of $\mathrm{y}(\mathrm{t}) . \mathrm{y}(\mathrm{t}-1)$ is the actual value at $\mathrm{t}-1$.

In this scenario contained within the article, the estimated value at $t$ is the predicted value of $y(t+1)$. So formula (5) is expressed as:

$\mathrm{s}(\mathrm{t})=\mathrm{a} * \mathrm{y}(\mathrm{t})+(1-\mathrm{a}) * \mathrm{~s}(\mathrm{t}-1)=\mathrm{a} *(\mathrm{y}(\mathrm{t})-\mathrm{s}(\mathrm{t}-1))+\mathrm{s}(\mathrm{t}-1)$.

$\mathrm{s}(\mathrm{t}-1)$ is the estimated value of $\mathrm{y}(\mathrm{t})$ in formula (6). The uncertainty of estimate will affect the estimated value of the next time. Formula (6) represents that application at $t$ is equal to queue length at $\mathrm{t}+1$. 
Comparing (5) to (6), this paper puts forward the third resource application program. Adding a smoothing factor dif can reduce the fluctuation of application to control the fluctuation of queue length and efficiency of the system, the dif is shown as:

$\operatorname{dif}=\mathrm{r} *(\mathrm{~s}(\mathrm{t}-1)-\mathrm{y}(\mathrm{t}))$.

$\mathrm{s}(\mathrm{t})=\mathrm{y}(\mathrm{t})+\operatorname{arr}-\mathrm{s}(\mathrm{t}-1)+\mathrm{dif}=(1-\mathrm{r}) * \mathrm{y}(\mathrm{t})+\operatorname{arr}-(1-\mathrm{r}) *(\mathrm{t}-1)$.

Let $\mathrm{a}=1-\mathrm{r}$. Then the final application bandwidth raised in this paper should be:

$\mathrm{s}(\mathrm{t})=\mathrm{a}^{*} \mathrm{y}(\mathrm{t})+$ arr $-\mathrm{a}^{*} \mathrm{~s}(\mathrm{t}-1)$.

$\mathrm{s}(\mathrm{t})$ is the application bandwidth at $\mathrm{t} . \mathrm{y}(\mathrm{t})$ is the queue length at $\mathrm{t} . \mathrm{s}(\mathrm{t}-1)$ is the application bandwidth at $\mathrm{t}-1$ and the estimated value of $\mathrm{y}(\mathrm{t})$.

The second item arr is the estimated value of the amount of packets arriving from $t$ to $t+1$. Through the business forecast $\mathrm{t}^{[14][15]}$, applying slot resource for business which may arrive during next frame in advance can reduce delay of business. The business forecasting method used in this paper is the local linear approximation (LLA $)^{[8]}$. The number of packets of the plane arriving in the $(\mathrm{t}+1)$-th request cycle is $N_{t+1}$, b is weighting factor.

$$
\begin{aligned}
& N_{t+1}=N_{t}+\mathrm{b}^{*} \Delta N_{t}-(1-\mathrm{b})^{*} \Delta N_{t}{ }^{\prime} . \\
& \Delta N_{t}=N_{t}-N_{t-1} . \\
& \Delta N_{t}{ }^{\prime}=\Delta N_{t}-\Delta N_{t-1} .
\end{aligned}
$$

Using application smoothing strategy proposed in this paper can avoid large fluctuation of queue length caused by excessive and too small application, which will greatly reduce the delay of business busy queue and prevent excessive loss when the queue is too long.

\section{Simulation}

This paper use MATLAB to simulate. The network structure of aeronautical broadband communication system between space and ground is shown in Fig.6. The simulation set ten planes and a base station.

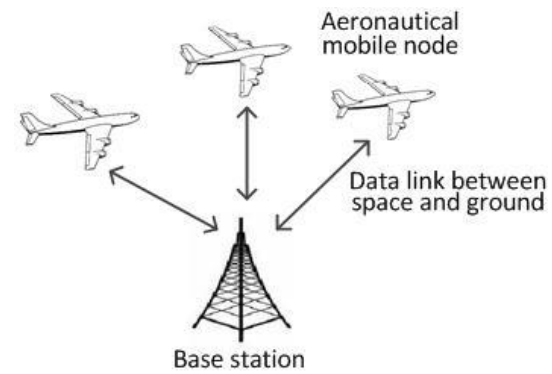

Fig. 6. Broadband communication system network structure between space and ground

\subsection{Frame Structure}

Plane terminal takes a multiframe as a unit in Fig.2. A multiframe includes DC and data. DC part sends control information and data part sends data packet. A multiframe is $58.32 \mathrm{~ms}$. A time slot is $0.72 \mathrm{~ms}$ and has 2 piles. A multiframe is divided into 81 slots (162 piles). Ten planes accounted for 10 piles of DC. The rest 152 piles are data frame. This article assumes that each pile transmits a packet. Frame period $\mathrm{T}_{\mathrm{mf}}$ and resource application allocation cycle $\mathrm{T}_{\mathrm{sr}}$ in Fig.2 are equal to $58.32 \mathrm{~ms}$.

\subsection{Business Source}

The business source is based on business model whose arrival process is time variant poisson distribution. In the complex wireless network, business has great inhomogeneity, uncertainty and paroxysmal character. Time-varying poisson process is necessary ${ }^{[12]}$.

Suppose packet arrival process of the plane follows the distribution of time-varying poisson process whose mean value is $\lambda_{n}(\mathrm{t})$. Different plane has different arrival rate. Fig. 7 show changes of $\lambda_{n}(\mathrm{t})$.

\subsection{Distribution Mode}

Referring to the existing satellite network resource allocation scheme, this paper uses the proportional allocation method for the radio resource management module of the base station ${ }^{[13]}$.

$$
v_{i}=\left\{\begin{array}{lr}
u_{i} & \text { if } \sum_{i=1}^{N} u_{i} \leq C \\
\frac{C}{\sum_{i=1}^{N} u_{i}} * u_{i} & \text { therwise }
\end{array} .\right.
$$


$u_{i}$ is request bandwidth of user i. $v_{i}$ is allocated bandwidth for user i.C is the total bandwidth.

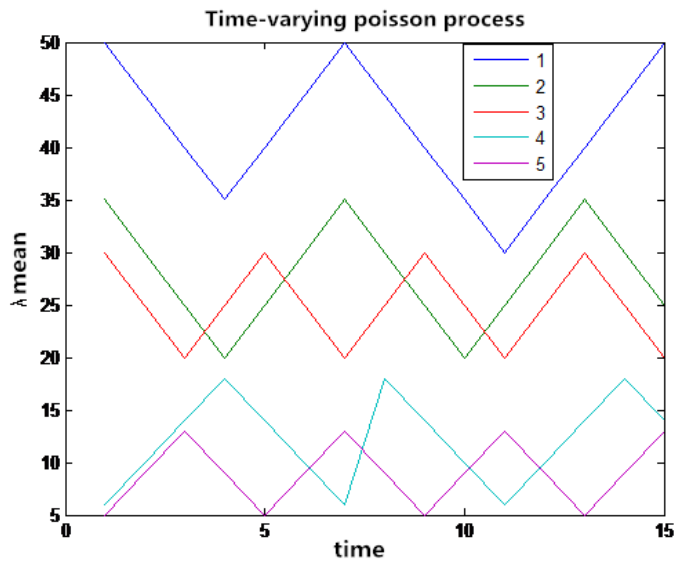

Fig. 7a. Mean value of plane 1 to 5

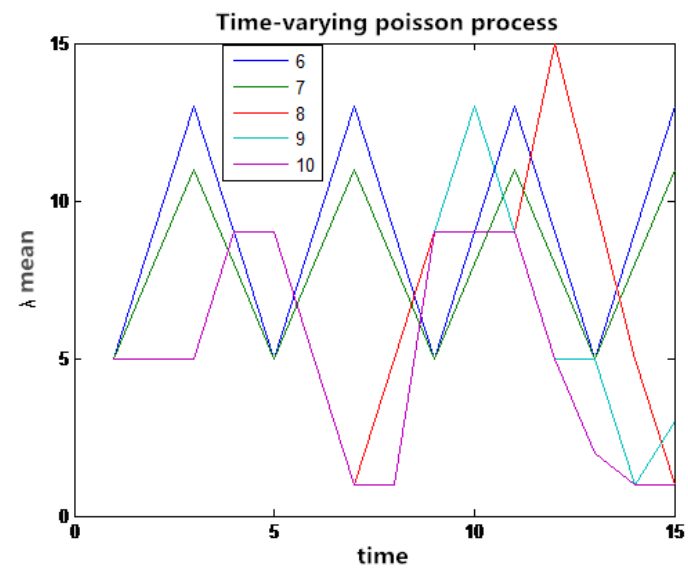

Fig. $7 \mathrm{~b}$. Mean value of plane 6 to 10

\section{Results and Analysis}

This paper mainly simulates the algorithm with delay ${ }^{[17]}$ and packet loss rate ${ }^{[16]}$. It compares the algorithm proposed in this paper (Algorithm 3), the algorithm based on queue length and control ${ }^{[8]}$ (Algorithm 1) and the algorithm based on base station feedback control ${ }^{[11]}$ (Algorithm 2).

Network delay in this paper is the time from resource application to allocation which is shown in Fig.3. Packet loss rate refers to the ratio of packet loss number and total packets which have entered the system in the entire simulation process. Packet loss rate is shown in Fig.2.

Application amount of algorithm 1, algorithm 2, algorithm 3 are shown in formula (1), (2), (9). a is 0.55 in (9).

In the condition of different network load degree, comparisons of delay and throughput property are shown in Fig.8. The contrast parameter in Fig.8a is the average time delay of the busiest user. The busiest user is the plane in Fig.7a whose business is the most. The busiest user requires handling the most business and it is often the most important user. So we need to improve the efficiency of this user. The contrast parameter in Fig. $8 \mathrm{~b}$ is the average time delay of user. It is the the average time delay of 10 planes. The contrast parameter in Fig. $8 \mathrm{c}$ is the packet loss rate of the busiest user. Because the queue length of other less busy users almost not reach the maximum amount, we only count the busiest user's packet loss rate. A low packet loss rate will support a high quality of service.

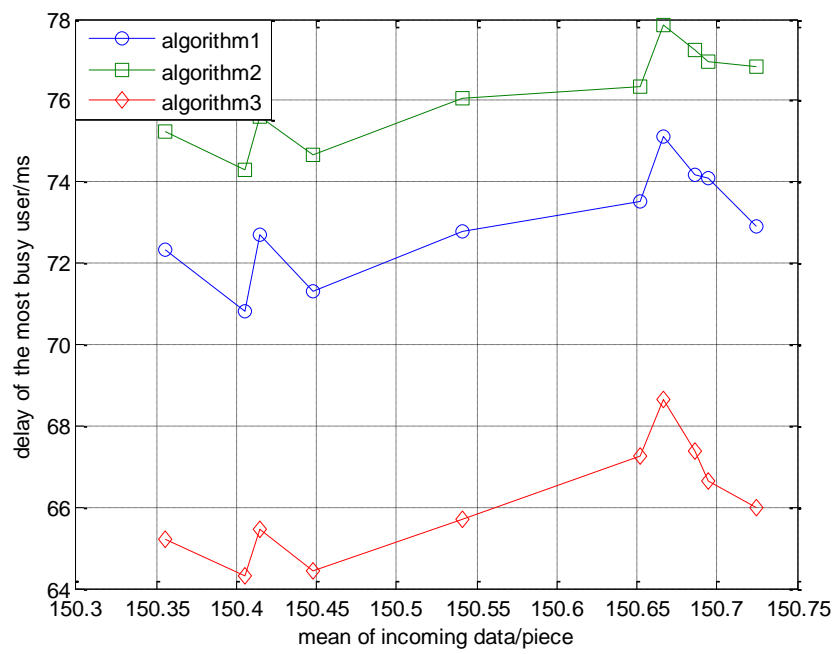

Fig. 8a. Delay of the busiest user

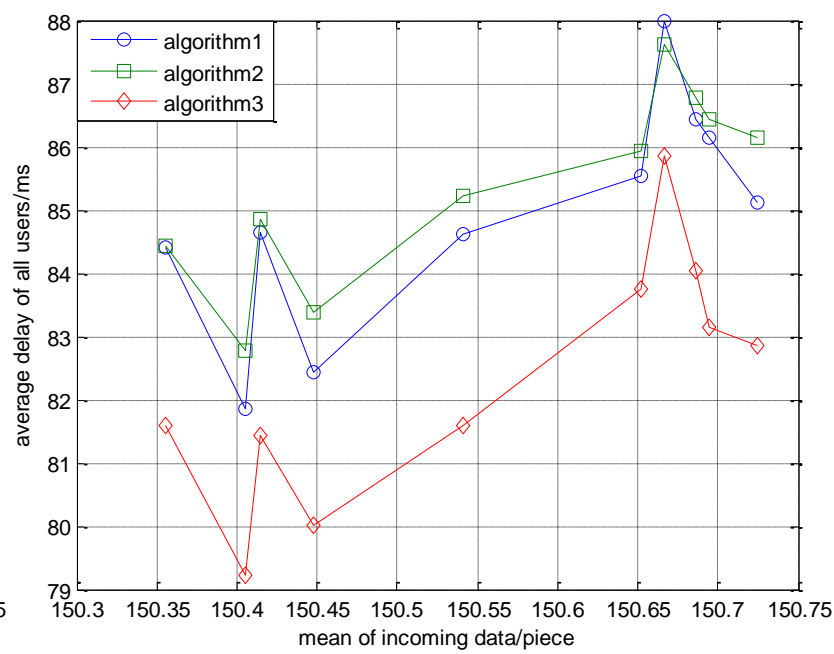

Fig. 8b. Average delay of all users 


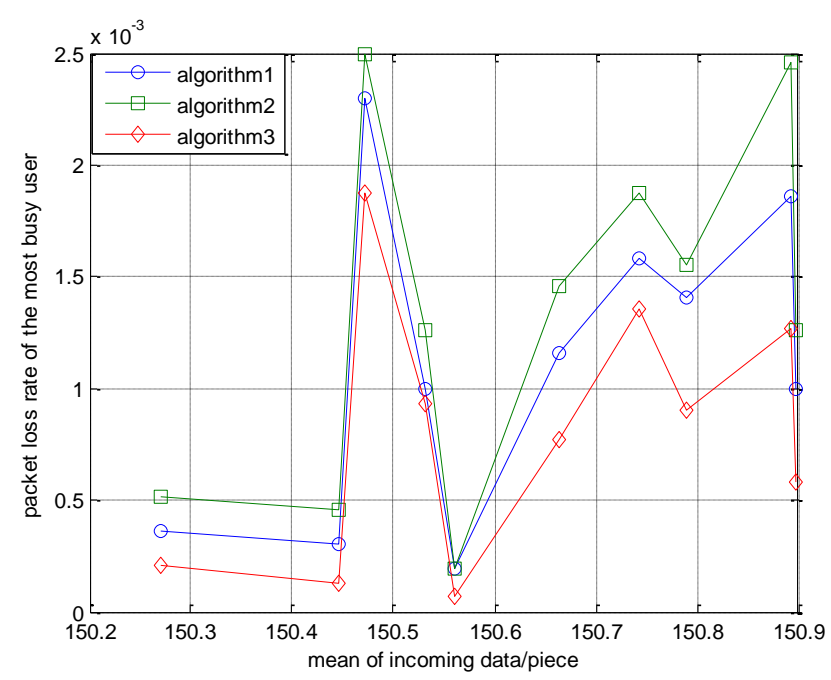

Fig. 8c. Packet loss rate of the busiest user

Time delay increases with the increase of network load from Fig.8a and Fig.8b. Under conditions of high load degree, the probability of network congestion increases. So the network service needs to wait longer.

Fig. $8 \mathrm{a}$ is average delay of the busiest queue. The delay of algorithm3(this paper) decreased about $10 \%$ compared to algorithm 1 and $15 \%$ compared to algorithm 2 . Because algorithm 3 adds a smoothing factor, the system can adjust whether the prediction is more or less at last frame. If predictive value is larger than the actual queue length, increase the amount of application of next frame to make the allocation not be too small. If predictive value is smaller than actual queue length, reduce the amount of application of next frame to avoid waste of resource caused by user's greed. This strategy enables the application of long and short queue to be stable and reduces the beneficial effect to short queue. The long queue's growth trend of queue length is less than the other two. Peak queue length reduced and delay dropped.

Fig. $8 \mathrm{~b}$ is the average delay of all users. The delay of algorithm3(this paper) decreased about $5 \%$ compared to algorithm1 and algorithm2. A significant reduction in time delay of the busiest user reduced the time delay of large part of business and raised the time delay of small part of business. So the average delay decreased.

Fig.8c is the packet loss rate of the busiest queue. The packet loss rate of algorithm3(this paper) decreased about $22 \%$ compared to algorithm 1 and $41 \%$ compared to algorithm 2 . The application amount of busiest user tends to be stable after the adjustment of the algorithm proposed in this paper. User can get the resource on the ground in time and business in the queue can be processed in time, so the length of the queue will not fluctuate great and the situation of queue length being greater than the maximum value will not appear frequently.

The simulation results show that under the LDACS1 protocol, the algorithm based on feedback control and exponential smoothing proposed in this paper can decrease time delay and packet loss rate, which can support a higher quality of service.

\section{Conclusion}

Aiming at the lack of existing wireless resource application algorithm, this paper proposed an application algorithm based on the combination of feedback control and exponential smoothing prediction. Smoothing factor can smooth the application and reduce the delay caused by the queue's large fluctuation. It can also reduce the resource waste caused by beneficial effect to short queue and increase throughput. Using business prediction algorithm to estimate the network traffic of mobile node can sufficiently reduce the negative impact on network resource application caused by long delay and accelerate the resource allocation time. Simulation results show that the performance of algorithm proposed in this paper is much better than the existing resource application algorithm. Under different degrees of load, the algorithm has good adaptability. 


\section{Acknowledgments}

This work was supported by the National Nature Science Foundation of China (No. 61171069, 61231013).

\section{References}

[1] Guo Jing. Current situation and development of air ground data link system of China Civil Aviation [J]. China Civil Aviation,2013,11:26-27.

[2] EUROCONTROL. LDACS1 System Definition Proposal Deliverable[M]. D2. 2009.

[3] T. Graupl, and M. Ehammer, The LDACS1 Link Layer Design, Future Aeronautical Communications [C], InTech Europe, pp. 291-317, 2011.

[4] TOMKOS I, KAZOVSKY L, KITAYAMA K I. Next-generation optical access networks: dynamic bandwidth allocation, resource use optimization, and QoS improvements[J]. Network, IEEE, 2012,26(2):4-6.

[5] Cheng Geng,Xia Weiwei,Shen Lianfeng. Dynamic bandwidth allocation algorithm based on adaptive transmission rate [J]. Journal of communication,2014,05:25-32.

[6] Chen Jian,Wen Yingyou,Zhao Dazhe. Wireless resource application in broadband multimedia satellite communication system [J]. Journal of Northeastern University (Natural Science Edition),2008,29(8): 1091-1095.

[7] Li Dou,Ji Binghui,Wang Feng. Dynamic allocation scheme research of broadband DVB-RCS satellite access channel based on mixed pure prediction $[\mathrm{J}]$. Journal of electronics and information,2008,30(3): 607-611.

[8] Qin Yong,Zhang Jun,Zhang Tao. DVB-RCS broadband satellite MAC protocol based on bandwidth distribution on demands [J]. Journal of Astronautics,2010,03:838-844

[9] Tian Weizhen, Guo Li, Yu Jiang. Hybrid time slot allocation algorithm based on traffic prediction [J]. Computer application research,2014,11:3438-3441+3476.

[10]Feng Shaodong,Wang Fan,Zhang Gengxin. Design and Simulation of MAC protocol in broadband multimedia satellite system [J]. Journal of system simulation,2011,04:740-744.

[11]Liu Lei,Xie Jun,Hu Gu Yu,Tang Bin. A new BoD bandwidth allocation allocation algorithm [J]. computer science,2013,11:61-64.

[12]Han Xiao. Research on resource allocation for future wireless communication:The balance between resource utilization ratio and QoS guarantee [D]. Zhejiang University,2013.

[13] Myung H G, Lim J,Goodman D J.Single carrier FDMA for uplink wireless transmission[J].IEEE Vehicular Technology Magazine, 2006, 1(3): 30-38.

[14]Zhang Xun,Nie Chang. Analysis of future wireless communication service demand forecasting method [J]. Designing Techniques of Posts and Telecommunications, 2013,12:29-33.

[15] Si Yuan,Chen Xin,Liu Zongqi. LTEEnd to end delay boundary analysis of cellular networks with self similar traffic [J]. computer science,2015,02:70-75.

[16]Liu Bei. Research on radio resource management strategy based on business experience [D]. University of Science \& Technology China,2012.

[17]Hua Wang,Lars Dittmann. Downlink resource management for QoS scheduling in IEEE 802.16 WiMAX networks.[J]. Computer Communications,2010,33: 